OPEN ACCESS

Edited by: Abd El-Latif Hesham,

Assiut University, Egypt

Reviewed by:

Braulio Esteve-Zarzoso, University of Rovira i Virgili, Spain

Giorgia Perpetuini,

University of Teramo, Italy Patrizia Romano,

University of Basilicata, Italy

*Correspondence:

Shuyi Qiu

syqiu@gzu.edu.cn

Specialty section:

This article was submitted to

Food Microbiology,

a section of the journal

Frontiers in Microbiology

Received: 23 April 2020

Accepted: 10 August 2020

Published: 31 August 2020

Citation:

Wang C, Tang J and Qiu S (2020)

Profiling of Fungal Diversity and Fermentative Yeasts in Traditional

Chinese Xiaoqu.

Front. Microbiol. 11:2103 doi: 10.3389/fmicb.2020.02103

\section{Profiling of Fungal Diversity and Fermentative Yeasts in Traditional Chinese Xiaoqu}

\author{
Chunxiao Wang ${ }^{1}$, Jiadai Tang ${ }^{1,2}$ and Shuyi Qiu' ${ }^{1 *}$
}

' Province Key Laboratory of Fermentation Engineering and Biopharmacy, School of Liquor and Food Engineering, Key Laboratory of Plant Resource Conservation and Germplasm Innovation in Mountainous Region (Ministry of Education), Guizhou University, Guiyang, China, ${ }^{2}$ Department of Liquor Making Engineering, Moutai Institute, Renhuai, China

To increase the safety and quality of baijiu and rice wine in China, controlling the use of traditional Xiaoqu by studying the beneficial yeasts present has recently been considered. The fungal diversity of six Chinese Xiaoqu including five traditional and one commercial samples was investigated to screen fermentative yeasts with low yields of higher alcohols. A high throughput sequencing approach detected fifteen fungal species with relative abundance higher than $1 \%$, and displayed dissimilarities of fungal diversity among Xiaoqu samples. The 15 fungal species were composed of 11 filamentous fungi with Rhizopus arrhizus as the most common specie and four yeast species, containing Hyphopichia burtonii, Saccharomyces cerevisiae, Saccharomycopsis fibuligera, and Saccharomycopsis malanga. Classic culture-dependent approaches, including 5.8SITS-RFLP analysis and D1/D2 sequencing of the 26S rRNA gene, identified nine yeast species in the five traditional Chinese Xiaoqu. In addition to the four yeast species also detected by high throughput sequencing approach, the other five yeast species isolated were Clavispora lusitaniae, Cryptococcus neoformans, Komagataella pastoris, Trichosporon asahii, and Wickerhamomyces anomalus. Further micro-fermentations of rice wine were performed using 19 single yeast isolates, and after the fermentation of rice wine, higher alcohols and ethanol were analyzed by gas chromatography. Two yeast strains, Saccharomyces cerevisiae FBKL2.8022 and Wickerhamomyces anomalus FBKL2.8023, were found to have low yields of higher alcohols and could produce $11.70 \%$ vol and $7.10 \%$ vol ethanol separately. This study for the first time, to the best of our knowledge, explored the fungal resources in traditional Xiaoqu from different regions of Guizhou, China. The screened S. cerevisiae and W. anomalus strains could be used to establish specific starters to promote the standardization of the production of baijiu and rice wine.

Keywords: rice wine, baijiu, high throughput sequencing, Saccharomyces cerevisiae, Wickerhamomyces anomalus

\section{INTRODUCTION}

Xiaoqu is a traditional fermentation starter in China that utilizes rice as a raw material (Gou et al., 2015; Liu and Sun, 2018). Xiaoqu is named as such due to its smaller size compared to Daqu, and is similar to Nuruk in Korea and Koji in Japan in terms of their use in producing rice wine (Jin et al., 2017; Cai et al., 2018). Xiaoqu is widely used in South China to produce rice, herbal, chi aroma baijiu and rice wine (Liu and Sun, 2018; Chen et al., 2019). Guizhou, a southwestern province, is 
famous for sauce aroma baijiu as well as traditional rice wine and Xiaoqu. Xiaoqu as a fermentation starter, impacts the flavor and quality of baijiu and rice wine (Jin et al., 2017; Zhang et al., 2018; Chen et al., 2019). Therefore, investigating the fungal diversity and beneficial yeast in traditional Guizhou Xiaoqu is necessary to improve the quality of baijiu and rice wine in China.

The production methods of Xiaoqu stem from ancient times in China (Jin et al., 2017). Traditionally, Xiaoqu is produced from rice flour inoculated with $Q u m u$, which is mature Xiaoqu and has good amylolytic and fermentation properties (Figure 1). Qumu contains different fungi and bacteria, which originate from raw materials, production microhabitats, and the inoculation of molds and yeasts (Gou et al., 2015; Cai et al., 2018). Commercial Xiaoqu is generally inoculated with a pure culture of beneficial molds and yeasts, that are screened from traditional Qumu. However, some Guizhou distilleries still use homemade Qumu, known as traditional Xiaoqu, to maintain their distinctive flavors. Traditional Xiaoqu contains rich natural microbial resources, especially yeasts and molds that play key roles (Wu et al., 2017). A typical example is Rhizopus Q303, a mold screened from traditional Xiaoqu by the Guizhou Provincial Light Industry Scientific Research Institute in 1977 that has been widely applied in baijiu and rice wine production due to its strong amylolytic ability (Fu, 1987). Investigating the fungal diversity of traditional Xiaoqu is beneficial for the isolation of advantageous molds and yeasts, which traditionally relied on laborious culture-dependent approaches and has only recently been upgraded to high throughput sequencing techniques. The advantageous molds and yeasts isolated from traditional Xiaoqu include Rhizopus oryzae, Rhizopus peka, Rhizopus chinensis, Absidia sp., Aspergillus oryzae, Mucor rouxianus, Saccharomyces cerevisiae, Saccharomycopsis fibuligera and Wickerhamomyces anomalus (synonyms Hansenula anomala, Pichia anomala) (Wu, 2004; Li and Fang, 2007; Jin et al., 2017). Gou et al. (2015) identified two primary fungal species of traditional Xiaoqu from Sichuan province, R. oryzae and S'copsis fibuligera, using 18S-amplicon high throughput sequencing techniques. Four species Rhizopus stolonifer, Cunninghamella bertholletiae, $S$. cerevisiae, and Candida fennica were identified in traditional Xiaoqu from Hubei and Sichuan provinces by $18 \mathrm{~S}$-amplicon high throughput sequencing techniques (Wu et al., 2017). Cai et al. (2018) detected eight amylolytic species of Rhizopus, Aspergillus, Mucor, Neurospora, and Saccharomycopsis, and four ethanol-producing species of Saccharomyces, Pichia and Candida in traditional Xiaoqu from Hubei, Jiangsu, Zhejiang, Guangxi, Sichuan, and Guizhou provinces using ITS-amplicon high throughput sequencing techniques. However, limited information is currently available regarding fungal diversity and beneficial fungi in traditional Chinese Xiaoqu.

Higher alcohols, such as isobutanol and isoamyl alcohol, at moderate levels contribute to the flavor of many alcoholic drinks, whereas excess levels may be a health hazard (Lachenmeier et al., 2008; Styger et al., 2011; Zhu et al., 2016), and thus, the amount present in alcoholic drinks should be controlled (Jin et al., 2017; Li et al., 2017). Higher alcohols are secondary metabolites of yeast during alcoholic fermentation, and are primarily synthesized by the catabolism of amino acids, also known as the Ehrlich pathway (Hazelwood et al., 2008). Studies from China have involved the screening of several yeast strains with low yields of higher alcohols such as S. cerevisiae (Strain code JH301, AY15BAT2, and ARTP5, etc.) obtained by natural separation, haploid preparation, or strain mutagenesis (Wang et al., 2019a). To the best of our knowledge, no yeasts with low yields of higher alcohols have previously been isolated from Xiaoqu. Traditional rice wine production methods depend on the individual's operation experience and lack control and standardization, and thus, commonly cause headaches or hangovers due to excess amounts of higher alcohols and methanol present (Chung et al., 2012; Yan et al., 2012). Sake yeast (Saccharomyces sake) strains with favorable traits, such as high fermentation ability (Kyokai Strain No. 6) and non-urea-producing (Kyokai Strain No. 1901), have been used for sake brewing since 1895, demonstrating the role of beneficial yeast on sake fermentation and quality improvement (Ohya and Kashima, 2019). Therefore, using the rich fungal resources in traditional Chinese Xiaoqu to screen fermentative yeasts with low yields of higher alcohols is advantageous.

To promote standardization of the production of baijiu and rice wine from Xiaoqu, beneficial molds and yeasts with specific industrial use have been a focus for producing starters. The aim of this study was first to collect traditional Xiaoqu from different regions in the Guizhou province of China and analyze their fungal resources. Secondly, this study aims to screen yeasts with ethanol-producing abilities and low yields of higher alcohols on the basis of the rich fungal resources in traditional folk Xiaoqu. Fungal diversity in Xiaoqu samples was investigated via high throughput sequencing techniques. Yeasts were isolated and identified through classic culture-dependent approaches. Microfermentations inoculated with a single isolated yeast species were performed to produce rice wine. The ethanol and higher alcohol contents after rice wine fermentation were analyzed, and fermentative yeasts were screened for their fermentation performance and higher alcohol yields.

\section{MATERIALS AND METHODS}

\section{Xiaoqu Samples}

Xiaoqu samples were collected from six distilleries in different regions of Guizhou of China in September 2017, with five samples being traditional Xiaoqu and one being commercial Xiaoqu. The six samples were labeled N, S, SW-PY, SW-XL, SW-XX, and $\mathrm{W}$ according to the location of the distilleries (Figure $\mathbf{1}$ and Supplementary Table S1). Xiaoqu samples were maintained on ice during transportation, and were stored at $-20^{\circ} \mathrm{C}$ for high throughput sequencing analysis and $4^{\circ} \mathrm{C}$ for yeast isolation.

\section{High Throughput Sequencing Analysis}

Six Xiaoqu samples (more than three blocks in each sample) were sent to Biomarker Technologies Corporation (Beijing, China) for DNA extraction and further high throughput sequencing. DNA was extracted from $0.25 \mathrm{~g}$ Xiaoqu samples using a PowerSoil $^{\circledR}$ DNA Isolation Kit (MO BIO Laboratories, Inc., Carlsbad, California). Primers of VnF 


\section{A The production procedures of Xiaoqu}
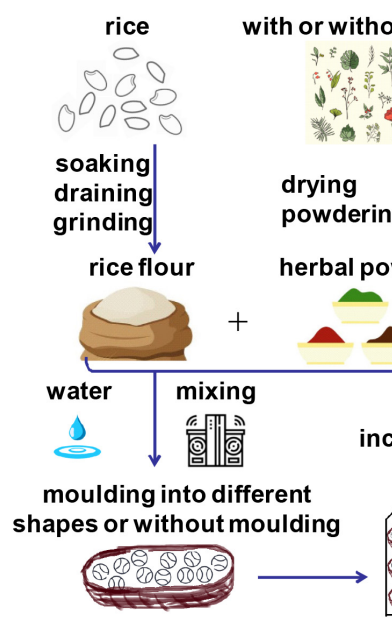

with or without herbs

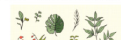

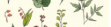

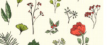

(2) $\%$

wdering $\square$
Qumu (mature Xiaoqu)

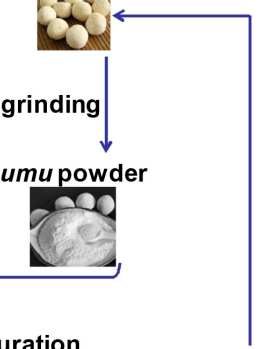
cubation, maturation and storage
B The shapes of six Xiaoqu samples

N

S
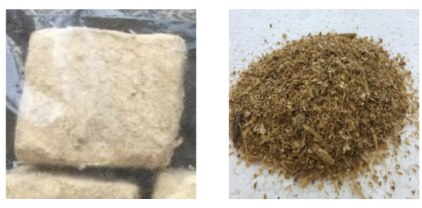

sW-XX

SW-XL
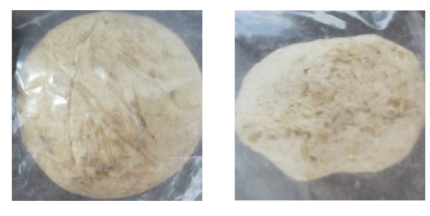

SW-PY

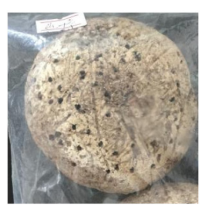

W

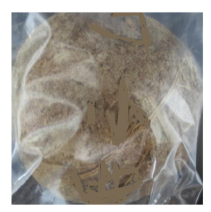

FIGURE 1 | The production processes of Xiaoqu and the shapes of six Xiaoqu samples. (A) The production processes of Xiaoqu. The primary raw material is rice, and others also include cereal or Chinese herbs. Rice is soaked in water for 2-3 h then drained and ground into rice flour. The rice flour with or without herbal powder is mixed with Qumu powder and water (60\%), then manually molded into different shapes (sphere, quadrate, cake-like, etc.). Sanqu was produced using an automatic starter-making disk machine without molding step (Wang et al., 2018). Xiaoqu was stored in a Qu house, namely a cultivation room, for approximately 4 days at $28-34^{\circ} \mathrm{C}$. Mature Xiaoqu is obtained after drying, maturation, and storage. (B) The shapes of six Xiaoqu samples. Xiaoqu samples were labeled as N, S, SW-PY, SW-XL, SW-XX, and W, corresponding to their collection from northern, southern, southwestern (Panzhou country), southwestern (Liguan of Xingren country), southwestern (Xiaojiawan of Xingren country), and western region of the Guizhou province.

$\left(5^{\prime}\right.$-CTTGGTCATTTAGAGGAAGTAA- $\left.3^{\prime}\right)$ and VnR $\left(5^{\prime}-\right.$ GCTGCGTTCTTCATCGATGC-3') with barcodes were used to amplify the ITS1 region. The amplification system included $0.3 \mu \mathrm{M}$ of each primer, 40-60 ng template DNA, $1 \mu \mathrm{L}$ KODFX-Neo (TOYOBO, Osaka, Japan), $1 \times$ buffer and $0.4 \mathrm{mM}$ dNTPs. The amplification conditions were $95^{\circ} \mathrm{C}$ for $5 \mathrm{~min}$, followed by 15 cycles of $95^{\circ} \mathrm{C}$ for $1 \mathrm{~min}, 50^{\circ} \mathrm{C}$ for $1 \mathrm{~min}$, and $72^{\circ} \mathrm{C}$ for $1 \mathrm{~min}$, and a final step at $72^{\circ} \mathrm{C}$ for $7 \mathrm{~min}$ (Zang et al., 2018). The amplification products were mixed with VAHTS DNA clean beads (Vazyme, Nanjing, China) for purification, and further amplified by solexa PCR using $0.25 \mu \mathrm{M}$ of each primer (MPPI-a and MPPI-b), $10 \mu \mathrm{L}$ purified amplification products, and $1 \times$ Phusion (NEB, Ipswich, Massachusetts). The solexa PCR conditions were $98^{\circ} \mathrm{C}$ for $30 \mathrm{~s}$, followed by 10 cycles of $98^{\circ} \mathrm{C}$ for $10 \mathrm{~s}, 65^{\circ} \mathrm{C}$ for $30 \mathrm{~s}$, and $72^{\circ} \mathrm{C}$ for $30 \mathrm{~s}$, and a final step at $72^{\circ} \mathrm{C}$ for $7 \mathrm{~min}$ (Zang et al., 2018). The solexa PCR products were used for DNA library construction after quantification by Qubit 2.0 (Thermo Fisher Scientific, Waltham, MA, United States), mix 1:1 by mass, and DNA gel extraction. Sequencing was performed based on the Illumina HiSeq 2500 platform. The obtained raw sequences were spliced and filtered, and chimeras were removed to generate effective sequences. The lengths of effective sequences were 100-500 bp depending on fungal species, and the average length was 207-284 bp among samples. The raw sequences of six samples analyzed by high throughput sequencing have been submitted to Sequence Read Archive (SRA) database ${ }^{1}$ with BioSample accessions SAMN14766771-SAMN14766776 under the BioProject PRJNA629089. The operational taxonomic units (OTUs) were generated with USEARCH software at a similarity

${ }^{1}$ https://submit.ncbi.nlm.nih.gov/subs/sra/ of $97 \%$, and RDP Classifier ${ }^{2}$ with a confidence threshold of 0.8 was used for taxonomic annotation based on the UNITE database $^{3}$. Alpha diversity was analyzed with Chao1, Shannon, and InvSimpson indexes using Mothur software.

\section{Yeast Isolation and Identification}

Each traditional Xiaoqu sample was sterilely ground into powder, and $10 \mathrm{~g}$ of Xiaoqu powder was diluted into $100 \mathrm{~mL}$ fluid with distilled water. The fluid with eight sterile glass beads with a diameter of $5 \mathrm{~mm}$ added was shaken at $150 \mathrm{r} / \mathrm{min}$ and $25^{\circ} \mathrm{C}$ for $20 \mathrm{~min}$. The decimal dilutions of the fluid were spread on WL nutrient agar (Hopebiol, Qingdao, China) in duplicate and incubated for 5 days at $28^{\circ} \mathrm{C}$. WL nutrient agar was used to collect all yeast with different morphologies from Xiaoqu samples due to its good identification characteristics (Pallmann et al., 2001). The plates with 30-200 yeast colonies present was selected for morphological observations. The total yeast concentration was quantified as $10^{8}-10^{9} \mathrm{cfu} / \mathrm{g}$ in each Xiaoqu sample. Colonies with different morphologies were selected from the plate, and each single colony was streaked onto new WL nutrient agar for purification and better classification. Pure yeast isolates were cultured in YPD broth (glucose $20 \mathrm{~g} / \mathrm{L}$, peptone $20 \mathrm{~g} / \mathrm{L}$, and yeast extract powder $10 \mathrm{~g} / \mathrm{L}$ ) at $28^{\circ} \mathrm{C}$ for 2 days, and further preserved with $20 \%$ (volume fraction) glycerol at $-80^{\circ} \mathrm{C}$.

All yeast isolates were cultured on potato dextrose agar (PDA) (BW-BIO, Shanghai, China) at $28^{\circ} \mathrm{C}$ for 2 days for further molecular analysis, including 5.8S-ITS-RFLP analysis with HaeIII restriction enzyme used and D1/D2 sequencing of

\footnotetext{
${ }^{2}$ http://sourceforge.net/projects/rdpclassifier/

${ }^{3}$ http://unite.ut.ee/index.php
} 
the 26S rRNA gene. The molecular analysis procedures, including DNA extraction, amplification, digestion, electrophoresis, and sequence alignment, followed those of a previous study by Wang et al. (2019b).

\section{Micro-Fermentation of Rice Wine With Single Yeast Species}

The milled round-grain glutinous rice used for microfermentation was purchased from Gaia Farm in northeastern China in 2018. The soluble protein content of the rice was analyzed via the Coomassie brilliant blue method (Cheng, 2014), the starch content was analyzed using the double-wavelength method (Jiang and Hu, 2013), and both were measured with Varioskan Flash (Thermo Fisher Scientific, Waltham, MA, United States). Fat and moisture content could be obtained during the starch analysis, because fat and moisture need to be removed from the rice for an accurate starch analysis.

The rice was developed into hydrolysate medium for microfermentation via the following procedures. First, $100 \mathrm{~g}$ of rice were soaked in $500 \mathrm{~mL}$ distilled water for $10 \mathrm{~h}$ then drained. The rice was transferred into a $500 \mathrm{~mL}$ triangle flask covered with air permeable sealing film and gauze, then sterilized at $121^{\circ} \mathrm{C}$ for $20 \mathrm{~min}$. After cooling, $150 \mathrm{~mL}$ sterile water, $70 \mathrm{U} / \mathrm{g}$ $\alpha$-amylase (Solarbio Life Sciences, Beijing, China), and $560 \mathrm{U} / \mathrm{g}$ glucoamylase (Solarbio Life Sciences, Beijing, China) were added and the solution was stirred then maintained in a water bath at $60^{\circ} \mathrm{C}$ for $30 \mathrm{~min}$. The $\mathrm{pH}$ of the rice hydrolysate medium was 6.5. A $500 \mathrm{~mL}$ triangle flask containing rice hydrolysate medium was used for micro-fermentation. The final volume of the rice hydrolysate medium in each triangle flask was $250 \mathrm{~mL}$. Yeast strains maintained as fresh colonies were pre-cultured in $30 \mathrm{~mL}$ YPD broth at $28^{\circ} \mathrm{C}$ overnight with a shaking speed of $120 \mathrm{r} / \mathrm{min}$. A hemocytometer was used under a microscope (Olympus, Tokyo, Japan) to quantify the yeast concentration in the YPD broth. A total of 19 yeast isolates were tested in the micro-fermentations as shown in Tables 2, 3. Each yeast isolate was inoculated into the rice hydrolysate medium at a concentration of $1 \times 10^{6}$ cells $/ \mathrm{mL}$. Then, $1 \mathrm{~mL}$ of fermentation broth diluted 1000 times after inoculation was spread on WL nutrient agar to check quantification and inoculation accuracy. Fermentations were performed in triplicate for each yeast species at $30^{\circ} \mathrm{C}$, and daily weight loss was recorded until not change on weight loss. The fermentation kinetics of 19 yeast isolates were shown in Supplementary Figure S1. The rice hydrolysate medium, after completing fermentation, was filtered with a single layer of gauze. Then, $100 \mathrm{~mL}$ filtrate was mixed with $100 \mathrm{~mL}$ distilled water and distilled in a $500 \mathrm{~mL}$ distilling flask until the distillate reached $100 \mathrm{~mL}$. The distillate was then sealed and stored at $4^{\circ} \mathrm{C}$ for further higher alcohols and ethanol analysis.

\section{Gas Chromatograph Analysis of Higher Alcohols and Ethanol}

The higher alcohols and ethanol in the distillate of rice wine were analyzed after filtration with a $0.22 \mu \mathrm{m}$ filter membrane. Analysis was performed with methanol as an external tags using GC-7890A (Agilent Appropriate Technology Co., Ltd., Santa
Clara, CA, United States) with a DB-FFAP capillary column (30 $\mathrm{m} \times 0.25 \mathrm{~mm} \times 0.25 \mu \mathrm{m}$ ) and flame ionization detector (Tang et al., 2019). Seven standard substances; ethanol, isoamyl alcohol, $\beta$-phenylethanol, isobutanol, propanol, hexanol, and butanol, were used to obtain the retention time and peak area of each substance at eight concentrations, as described by Tang et al. (2019). Further qualitative analysis was conducted according to the retention time, and quantitative analysis was conducted based on the produced standard curves. Temperature procedures were $45^{\circ} \mathrm{C}$ for $3 \mathrm{~min}$, heating to $120^{\circ} \mathrm{C}$ at the rate of $16^{\circ} \mathrm{C} / \mathrm{min}$, maintaining at $120^{\circ} \mathrm{C}$ for $3 \mathrm{~min}$, heating up to $220^{\circ} \mathrm{C}$ at the rate of $50^{\circ} \mathrm{C} / \mathrm{min}$, and holding at $220^{\circ} \mathrm{C}$ for $5 \mathrm{~min}$. Both the injector port and detector were maintained at $260^{\circ} \mathrm{C}$. The flow rates of air, hydrogen, and bypass were separately set as $300 \mathrm{~mL} / \mathrm{min}$, $30 \mathrm{~mL} / \mathrm{min}$, and $40 \mathrm{~mL} / \mathrm{min}$, and the total flow rate detected by GC-7890A was $44 \mathrm{~mL} / \mathrm{min}$. A $1 \mu \mathrm{L}$ sample was retrieved via a micro-syringe with a split ratio of 40:1. A PCA analysis was performed for the 19 yeast isolates using IBM SPSS Statistics 24.0 based on their yields of higher alcohols and ethanol.

\section{RESULTS}

\section{Fungal Diversity in Xiaoqu Samples by High Throughput Sequencing Analysis}

High throughput sequencing analysis obtained 55992-75857 effective sequences, and 25-126 observed species among samples (Table 1). The fungal diversity in six Xiaoqu samples was evaluated through alpha diversity indexes of high throughput sequencing analysis. SW-XX showed the highest species richness (Chao 1) with 126 species detected, whereas $\mathrm{N}$ with Chinese herbs added showed the lowest species richness with 25 species detected. SW-PY exhibited the best species evenness (Shannon and InvSimpson), whereas $\mathrm{N}$ showed the worst species evenness. In addition to the differences in alpha diversity among samples, six Xiaoqu samples showed variations in the relative abundance of fungal species detected (Figure 2). In total, 15 fungal species were detected with relative abundances greater than 1\%; Aspergillus cibarius, Aspergillus penicillioides, Hyphopichia burtonii, Monascus purpureus, Mucor indicus, Penicillium citrinum, Rhizopus arrhizus, Rhizopus microsporus, Rhizomucor pusillus, S. cerevisiae, S'copsis fibuligera, Saccharomycopsis

TABLE 1 | Alpha diversity indexes of six Xiaoqu samples in high throughput sequencing analysis.

\begin{tabular}{lccccc}
\hline $\begin{array}{l}\text { Xiaoqu } \\
\text { samples }\end{array}$ & $\begin{array}{c}\text { Effective } \\
\text { sequence } \\
\text { numbers }\end{array}$ & $\begin{array}{c}\text { The } \\
\text { number of } \\
\text { observed } \\
\text { species }\end{array}$ & Chao1 & Shannon & $\begin{array}{c}\text { Inv- } \\
\text { Simpson }\end{array}$ \\
\hline W & 65503 & 38 & 68.33 & 1.52 & 3.22 \\
SW-PY & 72249 & 53 & 58.60 & 1.86 & 4.00 \\
N & 75857 & 25 & 38.75 & 0.92 & 2.21 \\
S & 55992 & 63 & 82.50 & 1.46 & 2.62 \\
SW-XX & 74421 & 126 & 127.25 & 1.76 & 2.62 \\
SW-XL & 74795 & 37 & 46.43 & 1.02 & 2.54
\end{tabular}




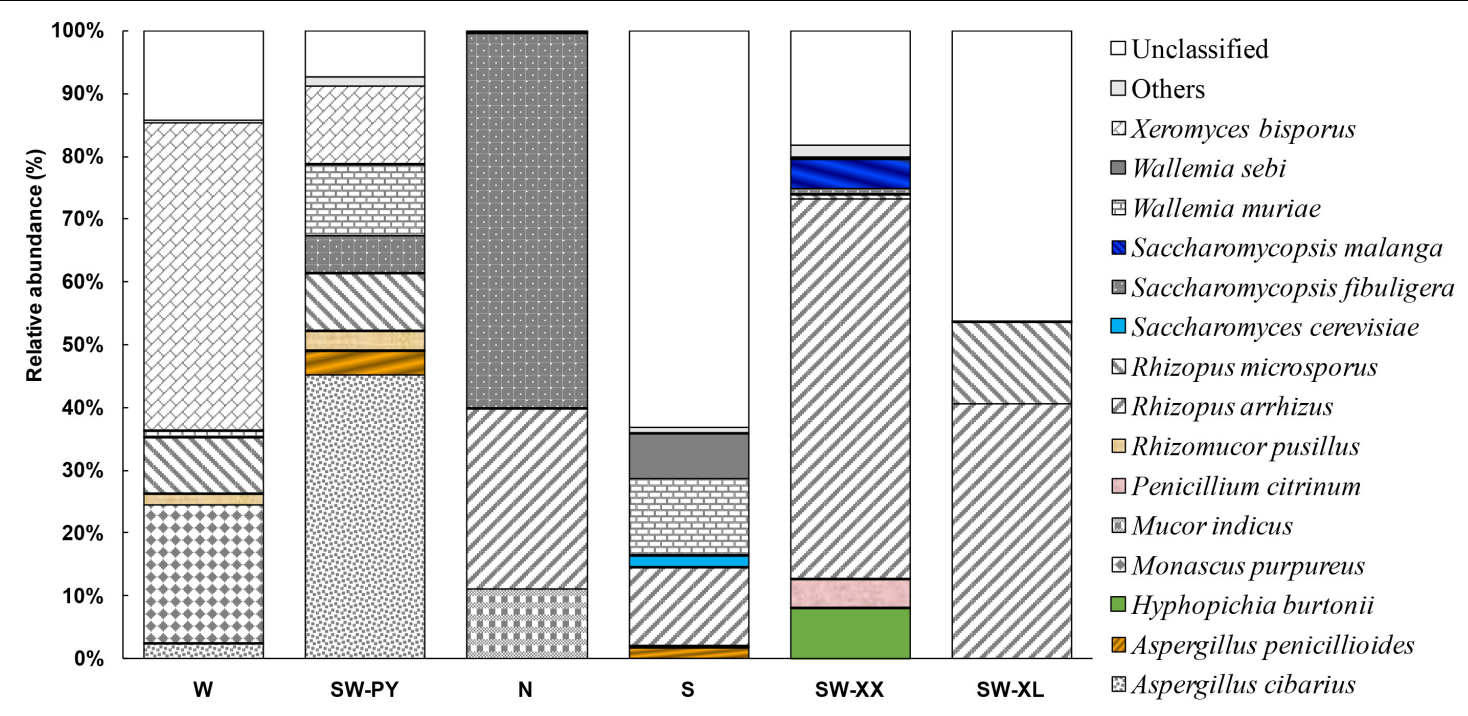

FIGURE 2 | Fungal species with relative abundance higher than 1\% in six Xiaoqu samples analyzed by high throughput sequencing approach.

malanga, Wallemia muriae, Wallemia sebi, and Xeromyces bisporus (Figure 2). R. arrhizus was the most common fungi specie detected from the six Xiaoqu samples, which appeared in four Xiaoqu samples with relative abundance higher than $10 \%$. And the next common fungi species were $R$. microsporus, $X$. bisporus, W. muriae, and S'copsis fibuligera existing in two or three Xiaoqu samples with relative abundance higher than $5 \%$. Unclassified species made up a proportion of species present, especially in S (63.1\%) and SW-XL (46.0\%). Most of the unclassified species could be classified at the genera level, and mainly belonged to the genera of Aspergillus and Wallemia. The unclassified Aspergillus species mainly existed in W (13.8\%), S (4.0\%), SW-XX (5.2\%) and SW-XL (45.9\%). And the unclassified Wallemia species mainly existed in SW-PY (6.9\%), and S (58.8\%). Noticeably, over $12 \%$ species in SW-XX could not be classified at the genera level, which contained $4.9 \%$ species belonging to unknown class of Ascomycota phylum, and 6.0\% belonging to unknown phylum.

Although high throughput sequencing analysis detected rich fungal resources, fewer yeasts were found than filamentous fungi in the six Xiaoqu samples. Only four yeast species were found to have a relative abundance higher than $1 \% ; H$. burtonii and S. cerevisiae was found in all samples except W, S'copsis fibuligera was found in all samples, and S'copsis malanga was only found in $\mathrm{S}$ and SW-XX. N with Chinese herbs added showed the highest relative abundance of yeast (59.8\% S'copsis fibuligera), and W showed the lowest relative abundance of yeast $(0.1 \%$ S'copsis fibuligera). Commercial Xiaoqu sample $\mathrm{S}$ was made without molding, and showed the highest proportion of $S$. cerevisiae (1.9\%) in the six Xiaoqu samples.

\section{Isolation and Identification of Yeasts in Traditional Xiaoqu Samples}

Although fewer yeasts were directly detected in the five traditional Xiaoqu samples by high throughput sequencing analysis, a quantity of yeasts could be isolated on WL nutrient agar. In order to dig out the potential target yeast species, the yeast colonies isolated were further purified and identified by 5.8S-ITS-RFLP analysis and D1/D2 sequencing of the $26 \mathrm{~S}$ rRNA gene. According to classic culture-dependent approaches, 62 yeast isolates were identified in nine species; Clavispora lusitaniae (one isolate), Cryptococcus neoformans (one isolate), $H$. burtonii (three isolates), Komagataella pastoris (one isolate), $S$. cerevisiae (eight isolates), S'copsis fibuligera (32 isolates), S'copsis malanga (nine isolates), Trichosporon asahii (one isolate), and Wickerhamomyces anomalus (six isolates). The sequence details of all isolates are described in Table 2. The nine yeast species showed seven different 5.8S-ITS-RFLP profiles, with the profile between $H$. burtonii and K. pastoris, and S'copsis fibuligera and $W$. anomalus being the same (Table 2).

The yeast concentration was $10^{8}-10^{9} \mathrm{cfu} / \mathrm{g}$ in Xiaoqu samples, with $\mathrm{N}$ containing the highest yeast concentration $\left(5.30 \times 10^{9}\right.$ $\mathrm{cfu} / \mathrm{g})$. S'copsis fibuligera was found in all the five traditional Xiaoqu samples (Supplementary Table S1); N (12 isolates), SWPY (11 isolates), SW-XL (4 isolates), W (4 isolates), SW-XX (1 isolates). The other eight yeast species were scattered in different Xiaoqu samples; SW-XX contained H. burtonii, K. pastoris, S'copsis malanga, and T. asahii, SW-XL contained C. lusitaniae, S. cerevisiae, and W. anomalus, SW-PY contained C. neoformans, and Xiaoqu samples $\mathrm{N}$ and $\mathrm{W}$ did not contain any other yeast species except S'copsis fibuligera.

\section{Screening of Yeasts With a Low Yield of Higher Alcohols}

The rice used for rice wine fermentations contained $9.21 \pm 0.03 \%$ (mass fraction) soluble protein, $0.67 \pm 0.12 \%$ fat, $14.6 \pm 0.20 \%$ moisture, $0.44 \pm 0.15 \%$ amylopectin, and $71.70 \pm 1.20 \%$ amylose. Six yeast species were selected for the micro-fermentation of rice wine, including eight $S$. cerevisiae, six $W$. anomalus, and two S'copsis malanga isolates, and one C. lusitaniae, 
TABLE 2 | Yeast species isolated from Xiaoqu samples.

\begin{tabular}{|c|c|c|c|c|c|}
\hline \multirow[t]{2}{*}{ Species/Sources } & \multicolumn{2}{|c|}{ 5.8S-ITS-RFLP analysis } & \multicolumn{3}{|c|}{ D1/D2 sequencing analysis of 26S rRNA gene } \\
\hline & $\begin{array}{c}\text { Size of PCR } \\
\text { products (bp) }\end{array}$ & $\begin{array}{l}\text { Size of Haelll } \\
\text { products (bp) }\end{array}$ & $\begin{array}{l}\text { Isolates } \\
\text { designations/GenBank } \\
\text { accession number of } \\
\text { isolates }\end{array}$ & $\begin{array}{c}\text { GenBank accession } \\
\text { number of aligned } \\
\text { strains }\end{array}$ & $\begin{array}{c}\text { Identity } \\
\text { percentage }\end{array}$ \\
\hline Clavispora lusitaniae/SW-XL & 350 & 350 & $\underline{\text { FBKL2.8010/MK722465 }}$ & EF063132 & $99 \%$ \\
\hline $\begin{array}{l}\text { Cryptococcus } \\
\text { neoformans/SW-PY }\end{array}$ & 600 & $500+120$ & FBKL2.8025/MK722477 & KY107157 & $99 \%$ \\
\hline Hyphopichia burtonii/SW-XX & 400 & 400 & $\begin{array}{l}\text { FBKL2.8018/MK722470, } \\
\text { FBKL2.8021/MK722473, } \\
\text { FBKL2.8062/MK722513 }\end{array}$ & U45712 & $99 \%$ \\
\hline Komagataella pastoris/SW-XX & 400 & 400 & FBKL2.8064/MK722515 & LC090848 & $100 \%$ \\
\hline$\frac{\text { Saccharomyces }}{\text { cerevisiae/SW-XL }}$ & 840 & $300+220+175+125$ & $\begin{array}{l}\frac{\text { FBKL2.8001-2.8004/ }}{\text { MK722456-722459, }} \\
\text { FBKL2.8008/MK722463, } \\
\text { FBKL2.8009/MK722464, } \\
\text { FBKL2.8012/MK722467, } \\
\text { FBKL2.8022/MK722474, }\end{array}$ & AY048154 & $99 \%$ \\
\hline $\begin{array}{l}\text { Saccharomycopsis } \\
\text { fibuligera/SW-XL, SW-XX, N, } \\
\text { SW-PY, W }\end{array}$ & 600 & 600 & $\begin{array}{l}\text { FBKL2.8026/MK722478, } \\
\text { FBKL2.8027/MK722479, } \\
\text { FBKL2.8029- } \\
\text { 2.8032/MK722481- } \\
\text { 722484, } \\
\text { FBKL2.8034- } \\
\text { 2.8059/MK722485-722510 }\end{array}$ & U40088 & $99 \%$ \\
\hline$\frac{\text { Saccharomycopsis }}{\text { malanga/SW-XX }}$ & 700 & 700 & $\begin{array}{l}\text { FBKL2.8015/MK722468, } \\
\text { FBKL2.8019/MK722471, } \\
\text { FBKL2.8020/MK722472, } \\
\text { FBKL2.8028/MK722480, } \\
\text { FBKL2.8060/MK722511, } \\
\text { FBKL2.8061/MK722512, } \\
\text { FBKL2.8063/MK722514, } \\
\text { FBKL2.8065/MK722516, } \\
\text { FBKL2.8066/MK722517 }\end{array}$ & U40135 & $99 \%$ \\
\hline Trichosporon asahii/SW-XX & 500 & 500 & $\underline{\text { FBKL2.8017/MK722469 }}$ & AF105393 & $99 \%$ \\
\hline$\frac{\text { Wickerhamomyces }}{\text { anomalus/SW-XL }}$ & 600 & 600 & $\begin{array}{l}\text { FBKL2.8005-2.8007/ } \\
\text { MK722460-722462, } \\
\text { FBKL2.8011/MK722466, } \\
\text { FBKL2.8023/MK722475, } \\
\text { FBKL2.8024/MK722476 }\end{array}$ & EF550341 & $99 \%$ \\
\hline
\end{tabular}

Species and isolates marked with underline were further analyzed in micro-fermentations.

H. burtonii, and T. asahii isolate each (Tables 2, 3). Microfermentations inoculated with the 19 isolates showed different fermentation periods (Supplementary Figure S1), ranging from 17 (C. Iusitaniae FBKL2.8010, S. cerevisiae FBKL2.8022, and $W$. anomalus FBKL2.8023) to 40 days (S. cerevisiae FBKL2.8003). The total higher alcohol contents in rice wine varied from 50.53 to $277.82 \mathrm{mg} / \mathrm{L}$, depending on the inoculated yeast strain (Table 3). Rice wine fermented by FBKL2.8019 contained the lowest amount of higher alcohols $(50.53 \mathrm{mg} / \mathrm{L})$, whereas rice wine fermented by FBKL2.8003 contained the highest amount (277.82 mg/L). Isoamyl alcohol, $\beta$-phenylethanol, isobutanol, and propanol are the four primary higher alcohols that were detected in rice wine. Isoamyl alcohol had the highest yield in most rice wines (19.30 mg/L to $120.57 \mathrm{mg} / \mathrm{L})$, followed by $\beta$-phenylethanol
(19.84 mg/L to $83.95 \mathrm{mg} / \mathrm{L})$, isobutanol (6.84 mg/L to $66.6 \mathrm{mg} / \mathrm{L})$, and propanol $(3.75 \mathrm{mg} / \mathrm{L}$ to $53.65 \mathrm{mg} / \mathrm{L})$. Most yeast strains produced similar levels of isobutanol and propanol in rice wine, which were nearly half that of isoamyl alcohol. The contents of butanol (not detected to $1.41 \mathrm{mg} / \mathrm{L}$ ) and hexanol $(0.72 \mathrm{mg} / \mathrm{L}$ to $9.10 \mathrm{mg} / \mathrm{L}$ ) were considerably lower (more than 10-fold) than the four primary higher alcohols identified in the rice wine (Table 3 ).

The ethanol contents in rice wine ranged from $2.12 \%$ to $11.70 \%$ (volume fraction), as shown in Table 3. Six S. cerevisiae strains (FBKL2.8001, FBKL2.8003, FBKL2.8008, FBKL2.8009, FBKL2.8012, and FBKL2.8022) and one $W$. anomalus strain (FBKL2.8006) were high ethanol producers with yields greater than 10\%. Two S. cerevisiae (FBKL2.8002 and FBKL2.8004) and five $W$. anomalus strains (FBKL2.8005, FBKL2.8007, 
TABLE 3 | The amount of higher alcohols and ethanol in rice wine fermented by 19 yeast isolates.

\begin{tabular}{|c|c|c|c|c|c|c|c|c|c|c|}
\hline Species/sources & Yeast isolates & $\begin{array}{l}\text { Fermentation } \\
\text { time (d) }\end{array}$ & $\begin{array}{c}\text { Ethanol } \\
\text { (\%vol) }\end{array}$ & $\begin{array}{l}\text { Propanol } \\
\text { (mg/L) }\end{array}$ & $\begin{array}{l}\text { Isobutanol } \\
\text { (mg/L) }\end{array}$ & $\begin{array}{l}\text { Butanol } \\
\text { (mg/L) }\end{array}$ & $\begin{array}{l}\text { Isoamyl } \\
\text { alcohol } \\
\text { (mg/L) }\end{array}$ & $\begin{array}{l}\text { Hexanol } \\
\text { (mg/L) }\end{array}$ & $\begin{array}{l}\beta \text {-phenylethanol } \\
\text { (mg/L) }\end{array}$ & $\begin{array}{l}\text { Sum of six higher } \\
\text { alcohols (mg/L) }\end{array}$ \\
\hline S'copsis malanga & FBKL2.8019 & 22 & $2.12 \pm 2.45$ & $3.75 \pm 4.51$ & $6.84 \pm 7.94$ & $0.00 \pm 0.00$ & $19.30 \pm 22.30$ & $0.80 \pm 0.92$ & $19.84 \pm 22.91$ & $50.53 \pm 58.42$ \\
\hline ISW-XX & FBKL2.8015 & 19 & $4.25 \pm 0.19$ & $25.65 \pm 2.65$ & $20.26 \pm 1.38$ & $0.01 \pm 0.01$ & $65.60 \pm 2.53$ & $1.41 \pm 0.05$ & $51.38 \pm 10.03$ & $164.31 \pm 7.02$ \\
\hline H. burtonii/SW-XX & FBKL2.8018 & 26 & $3.90 \pm 0.12$ & $4.72 \pm 0.72$ & $8.80 \pm 0.92$ & $0.00 \pm 0.00$ & $28.27 \pm 0.86$ & $8.81 \pm 13.11$ & $29.03 \pm 0.00$ & $79.63 \pm 13.90$ \\
\hline T. asahii/SW-XX & FBKL2.8017 & 22 & $3.84 \pm 0.18$ & $26.86 \pm 1.39$ & $17.39 \pm 1.09$ & $0.00 \pm 0.00$ & $57.91 \pm 3.54$ & $1.59 \pm 0.35$ & $35.69 \pm 7.72$ & $139.45 \pm 12.64$ \\
\hline \multirow[t]{6}{*}{ W. anomalus/SW-XL } & FBKL2.8007" & 34 & $7.76 \pm 0.37$ & $27.26 \pm 6.23$ & $22.16 \pm 4.46$ & $0.90 \pm 0.52$ & $58.83 \pm 11.74$ & $2.39 \pm 2.23$ & $46.65 \pm 0.78$ & $158.18 \pm 20.13$ \\
\hline & FBKL2.8024\# & 22 & $7.32 \pm 2.24$ & $28.98 \pm 7.24$ & $25.84 \pm 2.25$ & $1.10 \pm 0.16$ & $63.12 \pm 12.63$ & $0.72 \pm 0.46$ & $42.93 \pm 3.59$ & $162.68 \pm 25.38$ \\
\hline & FBKL2.8023" & 17 & $7.10 \pm 2.01$ & $30.29 \pm 8.31$ & $27.14 \pm 3.57$ & $1.36 \pm 0.23$ & $61.02 \pm 11.63$ & $0.90 \pm 0.03$ & $48.94 \pm 7.05$ & $169.66 \pm 27.30$ \\
\hline & FBKL2.8011 $\#$ & 38 & $8.38 \pm 0.13$ & $31.98 \pm 1.94$ & $28.30 \pm 0.59$ & $0.97 \pm 0.05$ & $66.31 \pm 0.97$ & $4.17 \pm 0.11$ & $48.09 \pm 1.68$ & $179.83 \pm 5.03$ \\
\hline & FBKL2.8005\# & 38 & $7.85 \pm 0.18$ & $28.56 \pm 1.58$ & $23.51 \pm 0.31$ & $0.88 \pm 0.07$ & $61.23 \pm 0.95$ & $4.15 \pm 0.76$ & $61.61 \pm 20.11$ & $179.95 \pm 20.00$ \\
\hline & FBKL2.8006* & 39 & $9.60 \pm 0.59$ & $31.47 \pm 9.76$ & $27.29 \pm 3.24$ & $0.59 \pm 0.30$ & $70.18 \pm 7.43$ & $5.52 \pm 0.33$ & $45.29 \pm 6.93$ & $180.35 \pm 27.93$ \\
\hline C. Iusitaniae/SW-XL & FBKL2.8010 & 17 & $5.22 \pm 0.11$ & $30.39 \pm 4.93$ & $40.08 \pm 1.61$ & $0.41 \pm 0.02$ & $82.72 \pm 3.50$ & $1.12 \pm 0.06$ & $58.16 \pm 2.40$ & $212.88 \pm 12.09$ \\
\hline \multirow[t]{8}{*}{ S. cerevisiae/SW-XL } & FBKL2.8004\# & 39 & $8.21 \pm 1.76$ & $38.51 \pm 9.33$ & $40.89 \pm 9.89$ & $0.96 \pm 0.26$ & $79.36 \pm 17.97$ & $5.28 \pm 0.24$ & $47.37 \pm 1.75$ & $212.36 \pm 35.81$ \\
\hline & FBKL2.8008* & 29 & $10.58 \pm 0.47$ & $43.37 \pm 2.48$ & $46.68 \pm 2.30$ & $1.23 \pm 0.07$ & $95.72 \pm 3.98$ & $6.03 \pm 2.01$ & $46.88 \pm 1.30$ & $239.90 \pm 8.80$ \\
\hline & FBKL2.8002 ${ }^{\#}$ & 28 & $9.22 \pm 0.31$ & $30.84 \pm 0.99$ & $46.06 \pm 0.77$ & $0.67 \pm 0.08$ & $81.75 \pm 6.29$ & $0.93 \pm 0.18$ & $83.72 \pm 22.79$ & $243.97 \pm 18.51$ \\
\hline & FBKL2.8009* & 39 & $10.23 \pm 0.14$ & $49.00 \pm 2.26$ & $46.80 \pm 0.66$ & $1.32 \pm 0.03$ & $92.19 \pm 1.93$ & $5.74 \pm 0.50$ & $49.32 \pm 0.89$ & $244.37 \pm 2.95$ \\
\hline & FBKL2.8001* & 36 & $9.91 \pm 1.14$ & $33.67 \pm 2.58$ & $53.01 \pm 10.56$ & $0.79 \pm 0.16$ & $95.37 \pm 16.05$ & $1.14 \pm 0.08$ & $72.98 \pm 17.50$ & $256.95 \pm 23.92$ \\
\hline & FBKL2.8012* & 38 & $11.29 \pm 0.35$ & $53.65 \pm 4.38$ & $50.92 \pm 2.66$ & $1.41 \pm 0.20$ & $102.72 \pm 5.75$ & $6.50 \pm 0.70$ & $42.33 \pm 4.25$ & $257.53 \pm 8.07$ \\
\hline & FBKL2.8022* & 17 & $11.70 \pm 0.19$ & $34.99 \pm 1.80$ & $66.60 \pm 2.81$ & $0.89 \pm 0.11$ & $120.57 \pm 2.29$ & $0.94 \pm 0.05$ & $53.77 \pm 1.06$ & $277.75 \pm 4.81$ \\
\hline & FBKL2.8003* & 40 & $9.96 \pm 0.23$ & $46.48 \pm 1.03$ & $46.61 \pm 5.51$ & $1.12 \pm 0.03$ & $90.57 \pm 6.28$ & $9.10 \pm 0.36$ & $83.95 \pm 8.36$ & $277.82 \pm 20.56$ \\
\hline
\end{tabular}

*Strains with ethanol yields greater than 10\%, "Strains with ethanol yields greater than $7 \%$ but lower than $10 \%$. 


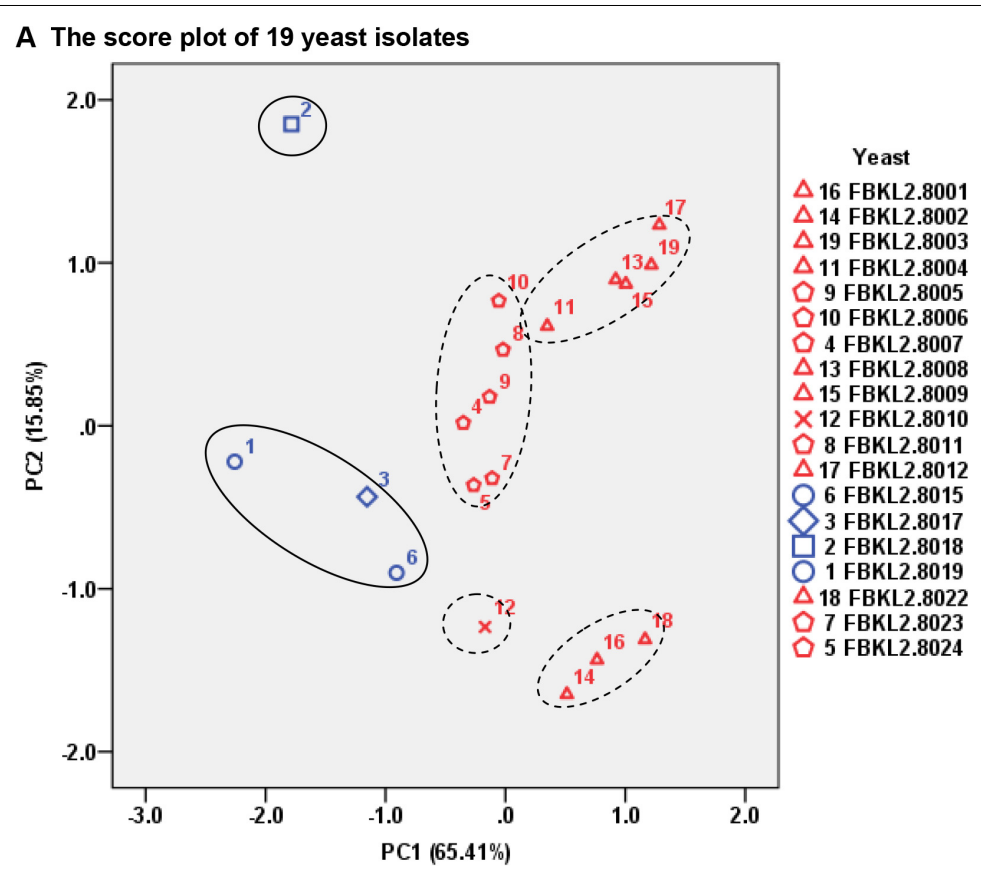

B The load diagram of seven factors

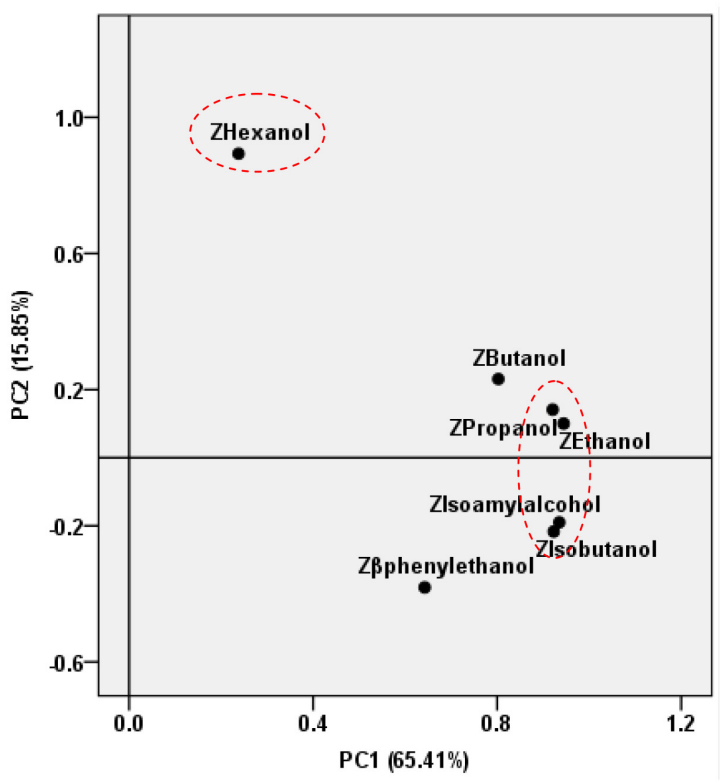

FIGURE 3 | PCA analysis of the 19 yeast isolates based on their yields of higher alcohols and ethanol in rice wine fermentation. (A) The score plot of 19 yeast isolates. The four yeast isolates with tags of $1,2,3$, and 6 originated from the Xiaoqu sample SW-XX. The other 15 yeast isolates originated from the Xiaoqu sample SW-XL. (B) The load diagram of seven factors.

FBKL2.8011, FBKL2.8023, FBKL2.8024) were medium ethanol producers with yields greater than $7 \%$, but lower than $10 \%$.

The PCA analysis differed the 19 yeast isolates into five groups, and the yeast isolates with different origins and identities were well differentiated under the influence of PC1 and PC2 (Figure 3A). PC1 was mainly affected by the contents of ethanol, isoamyl alcohol, propanol and isobutanol, and PC2 was mainly affected by the hexanol content (Figure 3B). Eight $S$. cerevisiae were separated into two groups under the influence of PC2, with FBKL2.8001, FBKL2.8002, and FBKL2.8022 being one group containing less hexanol content than the other groups with five isolates.

\section{DISCUSSION}

Increasing attention is being given to maintaining a high standardized quality of baijiu and other traditional Chinese alcoholic beverages such as rice wine, which requires a standardized and well-controlled production process. Therefore, in recent years, microbial diversity analyses in Jiuqu, the production environment, and fermentation processes have been undertaken to investigate microbial resources and potential beneficial microorganisms (Jin et al., 2017). The screened microorganisms are proposed to be safe and effective starters, as opposed to traditional starters, to promote the standardization of production (Cai et al., 2018). As well as a main baijiu and rice wine production region in China, Guizhou also traditionally produces Xiaoqu. To the best of our knowledge, this study, for the first time, analyzed fungal diversity among traditional Xiaoqu samples from different regions of Guizhou in China and further screened fermentative yeast with application value in the production of baijiu and rice wine.

Filamentous fungi were the primary fungi identified in six different Xiaoqu samples in this study, and several yeast species were detected with a relative abundance greater than $1 \%$, with unclassified fungi taking up a proportion of these species. These findings were consistent with those of previous studies regarding fungal diversity in Xiaoqu samples from different regions of Southern China, including provinces of Hubei, Jiangsu, Zhejiang, Guangxi, Sichuan, and East Guizhou (Gou et al., 2015; Wu et al., 2017; Cai et al., 2018). However, fungal diversity in Xiaoqu varied depending on the different geological locations. This study showed the obvious fungal diversity variation among Xiaoqu from six different regions of Guizhou province, even if two of the samples were from the same Country in the southwestern region of Guizhou. $R$. arrhizus was the most common filamentous fungi specie in Xiaoqu samples found in this study with the relative abundance of $28.8 \%$ in $\mathrm{N}$, $12.5 \%$ in S, $40.7 \%$ in SW-XL, and $60.4 \%$ in SW-XX, whereas a dominant occurrence of this species or genera was reported in Xiaoqu from Sichuan province (80.6\%, Gou et al., 2015), and Xiaoqu from Jiangsu, Zhejiang, Guangxi, and Guizhou provinces (higher than 95\%, Cai et al., 2018). In addition, Xiaoqu from neighboring provinces of Guizhou, also showed the variation of relative abundance of main fungal species, such as $80.6 \%$ of $R$. oryzae and $19.3 \%$ of S'copsis fibuligera in Xiaoqu from Luojiang Country of Sichuan province, 94.9\% 
of R. stolonifer in Xiaoqu from Pengzhou Country of Sichuan province, and $67.4 \%$ of $C$. bertholletiae, $18.9 \%$ of $R$. stolonifer, and $10.9 \%$ S. cerevisiae from Zigui Country of Hubei province (Gou et al., 2015; Wu et al., 2017; Cai et al., 2018). In addition to the difference of main fungal species, higher fungal species richness was exhibited in traditional Guizhou compared to other reported Xiaoqu. Both high throughput sequencing and classic culture-dependent approaches detected new fungal species that had not been reported in previous studies ( $\mathrm{Wu}, 2004$; Li and Fang, 2007; Gou et al., 2015; Jin et al., 2017; Wu et al., 2017; Cai et al., 2018), containing eight filamentous fungi species (M. purpureus, $P$. citrinum, R. arrhizus, $R$. microsporus, R. pusillus, W. muriae, W. sebi, and X. bisporus) and six yeast species (C. lusitaniae, C. neoformans, H. burtonii, K. pastoris, S'copsis malanga, and T. asahii).

The dissimilar fungal diversity among Xiaoqu in different geological locations might correlate with the production microhabitats of Xiaoqu, especially for the Qu house (Wang et al., 2020). Furthermore, the production technology of Xiaoqu such as the use of different raw materials and molding methods might also play a role in forming different fungal diversity (Wang et al., 2018). $\mathrm{N}$ had a variety of Chinese herbs added and exhibited the lowest species richness and evenness as well as the lowest proportion of unclassified species. $\mathrm{N}$ also showed the highest relative abundance of yeast (59.8\% S'copsis fibuligera). These findings confirmed the potential effect of Chinese herbs on microorganisms, of decreasing fungal diversity and promoting yeast growth (Wang et al., 2019a). Commercial Xiaoqu sample $\mathrm{S}$ had no molding (Sanqu) and showed the highest proportion of unclassified species (63.1\%, mainly from the genera of Aspergillus and Wallemia) and S. cerevisiae (1.9\%). These results differed from those of Wang et al. (2018) who found that traditional Xiaoqu showed higher bacterial diversity than Sanqu.

The combination of high throughput sequencing and culturedependent approaches helped to dig out the rich fungal resources in Xiaoqu in this study. The high throughput sequencing especially contributed to a comprehensive recognition of fungal diversity. The occurrence ratios of $H$. burtonii, S'copsis fibuligera, and S'copsis malanga analyzed by culture-dependent approaches coincided with their relative abundance in high throughput sequencing analysis. S. cerevisiae was found with relative abundance lower than $1 \%$ in N, SW-PY, SW-XL, and SW$\mathrm{XX}$ by high throughput sequencing analysis, whereas it was only isolated from SW-XL. The isolation and identification of new fungal species are beneficial for enriching fungal sequence databases, and thus, reducing the proportion of unclassified fungal species in the analysis of high throughput sequencing (Wang et al., 2019b). The unclassified species from the genera of Aspergillus and Wallemia, and from the unknown phylum or the unknown class of Ascomycota are needed to further analyze to explore the specific fungal resources in traditional Chinese Xiaoqu.

The content of higher-alcohols in California red wine was 140-417 mg/L (Zhu et al., 2016), in baijiu was about 600$1200 \mathrm{mg} / \mathrm{L}$ (Jin et al., 2017), and in European spirits was 0$10000 \mathrm{mg} / \mathrm{L}$ with the mean value of $4000 \mathrm{mg} / \mathrm{L}$ (Lachenmeier et al., 2008). Attempts have been made to reduce the amount of higher alcohols because of the potential health hazards and bitter taste caused by excess amount (Yan et al., 2012). This study for the first time evaluated the higher alcohols yields of wild yeast species in traditional Xiaoqu from Guizhou province, including two common yeast species in Xiaoqu, $S$. cerevisiae and $W$. anomalus, as well as four new yeast species, C. lusitaniae, H. burtonii, S'copsis malanga, and T. asahii. All yeast strains produced lower levels of higher alcohols in rice wine than other reported isolates $(0.34 \mathrm{~g} / \mathrm{L}$ to $3.98 \mathrm{~g} / \mathrm{L}$ ) (Wang et al., 2019a). However, the raw materials used are different in these studies including glutinous rice, corn, sorghum and malt extract medium, which contain different levels of amino acids, and thus, affect the yield of higher alcohols. Therefore, comparisons between previous studies and the present study would be improved if the same raw materials are used for fermentations of these isolates in future studies (Wang et al., 2019a). Moreover, S. cerevisiae ARTP5 obtained from mutagenesis has been found to produce $7.27 \mathrm{mg} / \mathrm{L}$ propanol, $14.14 \mathrm{mg} / \mathrm{L}$ isobutanol, and $44.99 \mathrm{mg} / \mathrm{L}$ isoamyl alcohol in fermented glutinous rice (Wang et al., 2019a). The laboratory S. cerevisiae ARTP5 produced less higher alcohols than the $S$. cerevisiae isolates from traditional Xiaoqu in Guizhou. Although the four new yeast species, C. lusitaniae, H. burtonii, S'copsis malanga, and T. asahii could produce less higher alcohols than most $S$. cerevisiae reported, their ethanol-producing ability was quite limited (lower than 5.5\%) which could not meet the demands of rice wine and baijiu. S. cerevisiae and $W$. anomalus displayed relatively strong ethanol-producing abilities of greater than $7 \%$, especially for $S$. cerevisiae strains, as reported by Fleet (2008). Therefore, S. cerevisiae FBKL2.8022 was screened as a fermentative yeast due to its high ethanol-producing ability (11.70\%), fast fermentation speed (17 days), and low yield of higher alcohols $(277.75 \mathrm{mg} / \mathrm{L})$. W. anomalus FBKL2.8023 has also been screened for making rice wine with medium levels of ethanol content as a result of its medium ethanolproducing ability (7.10\%), fast fermentation speed (17 days), and low yield of higher alcohols (169.66 mg/L). In addition, isoamyl alcohol and isobutanol were reported to be the two main higher alcohols in baijiu inoculated with Xiaoqu, the former taking up 45-65\% of the content of total higher alcohols (Yan et al., 2012). However, the rice wines fermented by 19 yeast isolates in this study showed a high content of $\beta$-phenylethanol, which was similar to isoamyl alcohol in some cases, and the content of propanol was also similar to isobutanol in this study.

$W$. anomalus is capable of producing phenylethyl acetate by the reaction of phenylethanol and acetic acid during fermentation (Liu et al., 2015). Our initial analysis demonstrated that $W$. anomalus ranked first on the tests of ester-producing ability in all the 66 yeast isolates (data not shown). Further studies should analyze the production ability of phenylethyl acetate of $W$. anomalus strains isolated from traditional Guizhou Xiaoqu samples, considering its phenylethanol yield. S'copsis fibuligera was the common yeast species in Xiaoqu and was largely isolated in this study. However, no isolates of S'copsis fibuligera were 
inoculated for micro-fermentations in this study, mainly because it was well known for the amylolytic ability instead of ethanolproducing ability (Cai et al., 2018). In addition, the colony and cell morphology of S'copsis fibuligera was similar to filamentous fungi, as well as the new yeast species of $C$. neoformans and $K$. pastoris, the ethanol-producing ability and higher alcohols yields of which were also not tested in this study. The esterproducing ability of the three yeast species were initially found lower than $W$. anomalus that we screened in this study (data not shown). Future studies will consider to use the mix inoculation of amylolytic, fermentative, and ester-producing fungal species to improve the aroma complexity of rice wine and baijiu.

In summary, traditional Chinese Xiaoqu samples showed different fungal diversity compared to another and the results of previous studies. The present study displayed distinct fungal resources for screening advantageous fungal species and producing standardized Xiaoqu. S. cerevisiae FBKL2.8022 and $W$. anomalus FBKL2.8023 were selected as fermentative yeasts with low yields of higher alcohols and high or medium ethanolproducing abilities. The amylolytic ability of fungal species, such as $R$. arrhizus, R. microsporus, S'copsis fibuligera, and S'copsis malanga, will be evaluated in future studies for mixed inoculation with the two yeast species screened. These studies will investigate the potential for their application in the production of baijiu and rice wine of high quality.

\section{DATA AVAILABILITY STATEMENT}

The datasets presented in this study can be found in online repositories. The names of the repository/repositories and accession number(s) can be found in the article/ Supplementary Material.

\section{REFERENCES}

Cai, H., Zhang, T., Zhang, Q., Luo, J., Cai, C., and Mao, J. (2018). Microbial diversity and chemical analysis of the starters used in traditional Chinese sweet rice wine. Food Microbiol. 73, 319-326. doi: 10.1016/j.fm.2018.02.002

Chen, S., Wang, C., Qian, M., Li, Z., and Xu, Y. (2019). Characterization of the key aroma compounds in aged Chinese rice wine by comparative aroma extract dilution analysis, quantitative measurements, aroma recombination, and omission studies. J. Agric. Food Chem. 67, 4876-4884. doi: 10.1021/acs.jafc. $9 \mathrm{~b} 01420$

Cheng, X. (2014). The Correlation Research between Nutritional Compositions in Chinese Rice Wine and Components in Raw Material of Glutinous Rice. Dissertation for the Degree of Master in Zhejiang A\&F University, Lin'an City.

Chung, H., Yoon, M. K., Kim, M., Park, S., Lee, J., and Kim, Y. (2012). Volatile hazardous compounds in alcoholic beverages. J. Appl. Biol. Chem. 55, 141-148. doi: $10.3839 /$ jabc.2012.022

Fleet, G. H. (2008). Wine yeasts for the future. FEMS Yeast Res. 8, 979-995. doi: $10.1111 / \mathrm{j} .1567-1364.2008 .00427 . x$

$\mathrm{Fu}$, J. (1987). The traits of Chinese Xiaoqu and main technical achievements. China Brew. 4, 14-17.

Gou, M., Wang, H. Z., Yuan, H. W., Zhang, W. X., Tang, Y. Q., and Kida, K. (2015). Characterization of the microbial community in three types of fermentation starters used for Chinese liquor production. J. Inst. Brew. 121, 620-627. doi: 10.1002/jib. 272

Hazelwood, L. A., Daran, J., van Maris, A. J. A., Pronk, J. T., and Dickinson, J. R. (2008). The Ehrlich pathway for fusel alcohol production: a century of

\section{AUTHOR CONTRIBUTIONS}

CW designed and performed the experiments and wrote the article. JT performed the experiments. SQ designed the article. All authors contributed to the article and approved the submitted version.

\section{FUNDING}

This study was supported by National Natural Science Foundation of China (31801523); Guizhou Province Science and Technology Project ([2019]2263; [2018]5781; [2016]25771); Construction Program of Biology First-class Discipline in Guizhou (GNYL[2017]009); Guizhou Education Bureau Research Program (KY[2018]120); and Guizhou University Talent Introduction Research Project ([2017]44).

\section{ACKNOWLEDGMENTS}

We would like to thank Editage (www.editage.cn) for English language editing.

\section{SUPPLEMENTARY MATERIAL}

The Supplementary Material for this article can be found online at: https://www.frontiersin.org/articles/10.3389/fmicb. 2020.02103/full\#supplementary-material

FIGURE S1 | The kinetic of micro-fermentation inoculated with 19 yeast isolates.

TABLE S1 | The details of Xiaoqu samples.

research on Saccharomyces cerevisiae metabolism. Appl. Environ. Microbiol. 74, 2259-2266. doi: 10.1128/aem.00934-938

Jiang, H., and Hu, Z. (2013). Determination of amylose and amylopectin content in indica rice by dual-wavelength spectrophotometry. Cereal Feed Ind. 2, 22-25.

Jin, G., Zhu, Y., and Xu, Y. (2017). Mystery behind Chinese liquor fermentation. Trends Food Sci. Technol. 63, 18-28. doi: 10.1016/j.tifs.2017.02.016

Lachenmeier, D. W., Haupt, S., and Schulz, K. (2008). Defining maximum levels of higher alcohols in alcoholic beverages and surrogate alcohol products. Regul. Toxicol. Pharmacol. 50, 313-321. doi: 10.1016/j.yrtph.2007. 12.008

Li, J., and Fang, J. (2007). Preliminary study on screening fungal $\alpha$-amylaseproducing strain and its solid-state fermentation conditions. Food Sci. 28, 373-378. doi: 10.3321/j.issn:1002-6630.2007.11.086

Li, W., Wang, J., Zhang, C., Ma, H., and Xiao, D. (2017). Regulation of Saccharomyces cerevisiae genetic engineering on the production of acetate esters and higher alcohols during Chinese Baijiu fermentation. J. Ind. Microbiol. Biotechnol. 44, 949-960. doi: 10.1007/s10295-017-1907-2

Liu, H., and Sun, B. (2018). Effect of fermentation processing on the flavor of Baijiu. J. Agric. Food Chem. 22, 5425-5432. doi: 10.1021/acs.jafc.8b00692

Liu, Z., Zhang, W., Chen, Y., He, D., and Ni, L. (2015). Synthesis pathway of phenethyl acetate in Wickerhamomyces anomalus. J. Chin. Inst. Food Sci. Technol. 15, 48-53. doi: 10.16429/j.1009-7848.2015.11.008

Ohya, Y., and Kashima, M. (2019). History, lineage and phenotypic differentiation of sake yeast. Biosci. Biotechnol. Biochem. 83, 1442-1448. doi: 10.1080/ 09168451.2018 .1564620 
Pallmann, C. L., Brown, J. A., Olineka, T. L., Cocolin, L., Mills, D. A., and Bisson, L. F. (2001). Use of WL medium to profile native flora fermentations. Am. J. Enol. Vitic. 52, 198-203. doi: 10.1016/S0065-2164(01)49013-7

Styger, G., Jacobson, D., and Bauer, F. F. (2011). Identifying genes that impact on aroma profiles produced by Saccharomyces cerevisiae and the production of higher alcohols. Appl. Microbiol. Biotechnol. 91, 713-730. doi: 10.1007/s00253011-3237-z

Tang, K., Zhou, H., Liu, C., Chen, S., Wang, X., Qiu, S., et al. (2019). Determination of 6 alcohols in rice wine by GC external standard method. China Brew. 38, 183-187. doi: 10.11882/j.issn.0254-5071.2019.04.036

Wang, C., Tang, J., Wu, X., Zhou, H., and Qiu, S. (2019a). Progress in research of functional microbes in Xiaoqu, a Chinese traditional fermentation starter for liquor and rice wine. Food Sci. 40, 309-316. doi: 10.7506/spkx1002-663020180901-004

Wang, C., Wu, C., and Qiu, S. (2019b). Yeast diversity investigation of Vitis davidii Föex during spontaneous fermentations using culture-dependent and highthroughput sequencing approaches. Food Res. Int. 126:108582. doi: 10.1016/j. foodres.2019.108582

Wang, C., Yuan, G., Su, W., Wang, X., and Qiu, S. (2020). Study on AcidProducing Microorganism From Qu-Making Environment and Mature qu in Baijiu. Food Science. Available online at: https://kns.cnki.net/KCMS/detail/11. 2206.ts.20191212.1332.066.html

Wang, J., Zhong, Q., Yang, Y., Li, H., Wang, L., Tong, Y., et al. (2018). Comparison of bacterial diversity between two traditional starters and the round-Koji-maker starter for traditional Cantonese Chi-flavor liquor brewing. Front. Microbiol. 9:1053. doi: 10.3389/fmicb.2018.01053

Wu, H., Zhang, S., Ma, Y., Zhou, J., Luo, H., and Yang, J. (2017). Comparison of microbial communities in the fermentation starter used to brew Xiaoqu liquor. J. Inst. Brew. 123, 113-120. doi: 10.1002/jib.388
Wu, X. (2004). Separation, purification and accreditation of Mold in Xiaoqu. Liquor Mak. Sci. Technol. 6, 33-34+36. doi: 10.13746/j.njkj.2004. 06.009

Yan, J., Fang, S., Jiang, W., Li, R., and Chen, M. (2012). Research on the reduction of fusel oil content in Xiaoqu liquor. Liquor Mak. 39, 32-35.

Zang, J., Xu, Y., Xia, W., Yu, D., Gao, P., Jiang, Q., et al. (2018). Dynamics and diversity of microbial community succession during fermentation of Suan yu, a Chinese traditional fermented fish, determined by high throughput sequencing. Food Res. Int. 111, 565-573. doi: 10.1016/j.foodres.2018. 05.076

Zhang, W., Cheng, Y., Li, Y., Du, G., Xie, G., Zou, H., et al. (2018). Adaptive evolution relieves nitrogen catabolite repression and decreases urea accumulation in cultures of the Chinese rice wine yeast strain Saccharomyces cerevisiae XZ-11. J. Agric. Food Chem. 66, 90619069.

Zhu, J., Shi, J. L., Lu, Y., Liu, L. P., and Liu, Y. L. (2016). Application of strains of Geotrichum spp. to decrease higher alcohols and to increase esters. J. Inst. Brew. 122, 147-155. doi: 10.1002/jib.287

Conflict of Interest: The authors declare that the research was conducted in the absence of any commercial or financial relationships that could be construed as a potential conflict of interest.

Copyright (C) 2020 Wang, Tang and Qiu. This is an open-access article distributed under the terms of the Creative Commons Attribution License (CC BY). The use, distribution or reproduction in other forums is permitted, provided the original author(s) and the copyright owner(s) are credited and that the original publication in this journal is cited, in accordance with accepted academic practice. No use, distribution or reproduction is permitted which does not comply with these terms. 